\title{
Channeled blast wave behavior based on longitudinal instabilities
}

\author{
M. Pohl ${ }^{1}$, I. Lerche ${ }^{2}$, and R. Schlickeiser ${ }^{1}$ \\ 1 Institut für Theoretische Physik, Lehrstuhl IV: Weltraum- und Astrophysik, Ruhr-Universität Bochum, \\ 44780 Bochum, Germany \\ 2 Department of Geological Sciences, University of South Carolina, Columbia, SC 29208, USA
}

Received 20 July 2001 / Accepted 22 November 2001

\begin{abstract}
To address the important issue of how kinetic energy of collimated blast waves is converted into radiation, Pohl \& Schlickeiser (2000) have recently investigated the relativistic two-stream instability of electromagnetic turbulence. They have shown that swept-up matter is quickly isotropized in the blast wave, which provides relativistic particles and, as a result, radiation. Here we present new calculations for the electrostatic instability in such systems. It is shown that the electrostatic instability is faster than the electromagnetic instability for highly relativistic beams. However, even after relaxation of the beam via the faster electrostatic turbulence, the beam is still unstable with respect to the electromagnetic waves, thus providing the isotropization required for efficient production of radiation. While the emission spectra in the model of Pohl \& Schlickeiser have to be modified, the basic characteristics persist.
\end{abstract}

Key words. instabilities - plasmas - turbulence - BL Lacertae objects: general

\section{Introduction}

Published models for the $\gamma$-ray emission of blazars are usually based on interactions of highly relativistic electrons of unspecified origin propagating in the relativistic jet of active galactic nuclei (Hartman et al. 1997; Bicknell et al. 2001; Catanese \& Weekes 1999). The usual shock or stochastic electron acceleration processes would have to be very fast to compete efficiently with the strong radiative losses at high electron energies, which operate on timescales of the order of ten seconds for the rapidly variable $\gamma$-ray blazars, which is similar to the duration of a single shock crossing cycle for $\mathrm{TeV}$ electrons at a moderately relativistic shock assuming Bohm diffusion (Gallant \& Achterberg 1998). Energetic electrons may also result as secondaries from photomeson production of highly relativistic hadrons, but the observed short variability timescale places extreme constraints on the magnetic field strength, for the hadron gyroradius has to be much smaller than the system size, and on the Doppler factor, for the intrinsic timescale for switching off the cascade is linked to the observed soft photon flux.

Here we consider the energisation of relativistic particles in jets by interactions with the surrounding medium. The rapid variability displayed in the $\gamma$-ray light curves of blazars requires the emission regions be less than $\sim 0.01 \mathrm{pc}$ in size. Also, VLBI observations of blazars indicate that

Send offprint requests to: M. Pohl, e-mail: mkp@tp4.ruhr-uni-bochum.de the jets are not continuously filled emission regions, but consist of individual structures which relativistically move along a common trajectory and can be followed over years. On larger scales optical data still show individual knots, but also emission in-between (e.g. Boksenberg et al. 1992). Detailed spectral studies (e.g. Meisenheimer et al. 1996) indicate that the spectrum of the synchrotron radiating electrons is fairly independent of position, suggesting that many acceleration sites with uniform characteristics are operational on scales of $\sim 100 \mathrm{pc}$.

Accounting for these findings we model a jet as a channeled outflow with relativistic bulk velocity $V$, consisting of isolated plasma clouds or blast waves which contain cold electrons and protons of density $n_{\mathrm{b}}$ (see Fig. 1). These clouds may correspond to the individual components observed in VLBI images of blazars, whereas many of them and secondary electrons of escaped nucleons would contribute to the optical and X-ray jets observed on larger scales in nearby AGN such as M 87. For convenience we assume that the outflow is directed parallel to the uniform background magnetic field. We also neglect a possible expansion of the jet cloud in this paper. Expansion is expected to occur on much longer timescales than the effects discussed here.

Each cloud of protons and electrons propagates into the surrounding interstellar medium that consists of cold protons and electrons of density $n_{i}^{*}$ (quantities indexed with an asterisk are measured in the laboratory (galaxy) frame). Viewed from the coordinate system comoving with 


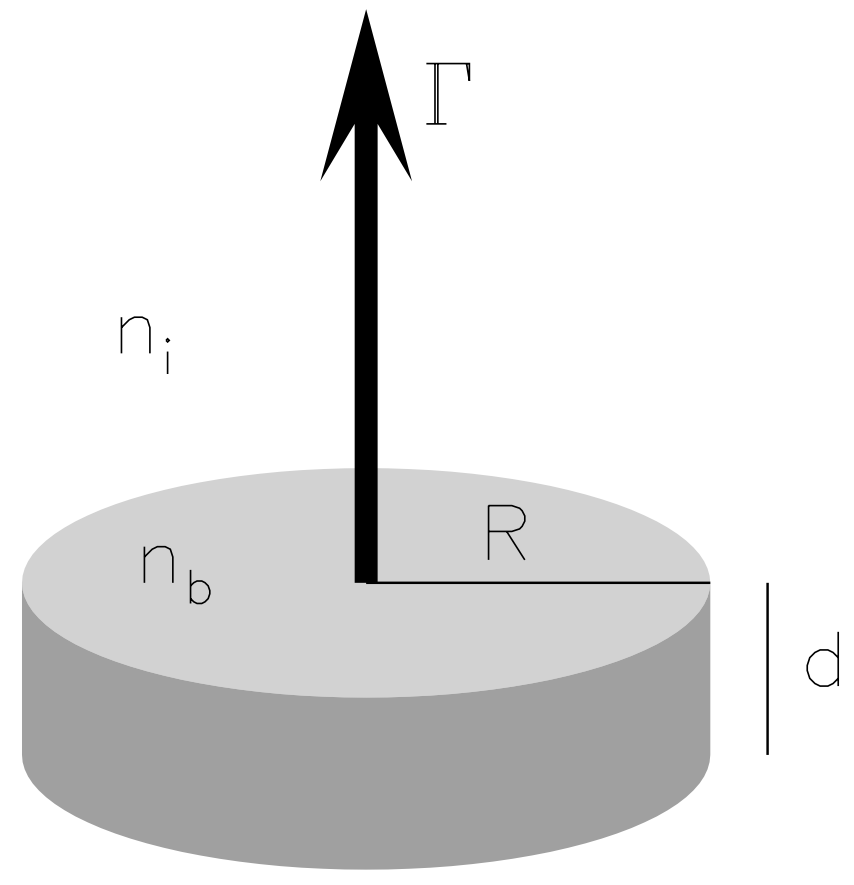

Fig. 1. Sketch of the basic geometry. The thickness of the channeled blast wave $d$, measured in its rest frame, is much smaller than its halfdiameter. The blast wave moves with a bulk Lorentz factor $\Gamma$ through ambient matter of density $n_{\mathrm{i}}$.

the outflow, the interstellar protons and electrons represent a proton-electron beam propagating with relativistic speed $-V$ antiparallel to the uniform magnetic field direction. This situation is unstable and waves will be excited which backreact on the incoming beam. In our earlier analysis (Pohl \& Schlickeiser 2000) the stability of this beam was examined under the assumption that the background magnetic field is uniform and directed parallel to the direction of motion. It was shown that the beam very quickly excites low-frequency electromagnetic waves, which quasilinearly isotropize the incoming interstellar electrons and protons in the blast wave plasma, thus providing relativistic particles. These processes are essentially those thought to operate in the first half-cycle of relativistic shock acceleration, but rather than assuming the existence of a hydrodynamical shock and a particular intensity of MHD turbulence, kinetic theory was used to self-consistently calculate the build-up of turbulence and its backreaction on the incoming interstellar particles.

The protons carry the bulk of the power, because velocities are conserved in the isotropization process and hence the protons have a factor of $\sim 2000$ more kinetic energy than the electrons. The protons produce high energy emission via the production of neutral pions in inelastic pp collisions. In parallel charged pions would provide secondary electrons which emit synchrotron radiation as well as bremsstrahlung and inverse Compton scattering. As discussed in Pohl \& Schlickeiser (2000), the high energy emission produced by these particles has characteristics typical of BL Lacertae objects. The observed secular variability of the $\gamma$-ray emission of AGN would be related to the existence (or non-existence) of a relativistic blast wave in the sources, and thus to the availability of free energy in the system. The observed fast variability, on the other hand, would be caused by density inhomogeneities in the interstellar medium through which the blast wave propagates.

In parallel to the $\gamma$-rays, neutrinos are emitted whose spectrum and flux would be closely correlated with those of the $\gamma$-rays, which permits one to use the $\gamma$-ray light curves of blazars to very efficiently search for neutrino emission as a diagnostic for an hadronic origin of the high energy radiation (Schuster et al. 2002).

In the initial stage virtually no radio emission would be produced, for the plasma frequency and the synchrotron self-absorption frequency would be too high. After the initial $\gamma$-ray emission phase, when the blast wave has decelerated and expanded, a mm-radio flare would build up, which behaviour is preferentially observed in radio to $\gamma$-ray correlation studies of EGRET sources (Mücke et al. 1996).

Here we expand on the previous treatment by calculating the two-stream instability for longitudinal, electrostatic waves. In contrast to electromagnetic waves, which scatter the particles in pitch angle but preserve their kinetic energy until the distribution is isotropized, the electrostatic waves change the particles' energy until a plateau distribution is established.

\section{Longitudinal instabilities of a proton-electron beam}

\subsection{The dispersion relation}

For charge $e$, mass $m$ particles under the action of an electrical field $\boldsymbol{E}=e_{\|} E_{\|} \exp (\imath k(x-a t))$, in the direction parallel to an ambient magnetic field the perturbations, $\delta f$, to the original distribution function satisfy

$\frac{\partial \delta f}{\partial t}+\boldsymbol{v} \frac{\partial \delta f}{\partial \boldsymbol{x}}+e \boldsymbol{E} \frac{\partial f}{\partial \boldsymbol{p}}=0$

which, to first order in $\delta f / f_{0}$, and with $\delta f$ also taken to vary as $\delta f(p) \exp (\imath k(x-a t))$ gives

$$
\begin{aligned}
& \imath k\left(v_{\|}-a\right) \delta f+e E_{\|} \frac{\partial f_{0}}{\partial p_{\|}}=0 \\
& \text { i.e. } \quad \delta f=\frac{\imath e E_{\|}}{k\left(v_{\|}-a\right)} \frac{\partial f_{0}}{\partial p_{\|}} .
\end{aligned}
$$

The electrostatic balance equation requires

$\operatorname{div} \boldsymbol{E}=4 \pi \sum e \int \delta f \mathrm{~d}^{3} p$

where the sum is over charge species. Thus

$k^{2} E_{\|}=4 \pi E_{\|} \sum e^{2} \int \frac{\frac{\partial f_{0}}{\partial p_{\|}}}{v_{\|}-a} \mathrm{~d}^{3} p$. 
Then the dispersion relation is

$k^{2}=4 \pi \sum e^{2} \int \frac{\frac{\partial f_{0}}{\partial p_{\|}}}{v_{\|}-a} \mathrm{~d}^{3} p$.

Let $a=a_{\mathrm{R}}+\imath a_{\mathrm{I}}$. Then split the RHS of Eq. (6) formally into the real part, $J$, and the imaginary part, $I$,

$k^{2}=J\left(a_{\mathrm{R}}, a_{\mathrm{I}}\right)+\imath I\left(a_{\mathrm{R}}, a_{\mathrm{I}}\right)$.

Because $k^{2}$ is meromorphic, then

$I\left(a_{\mathrm{R}}, a_{\mathrm{I}}\right)=0 \simeq I\left(a_{\mathrm{R}}, 0\right)+\left.a_{\mathrm{I}} \frac{\partial I\left(a_{\mathrm{R}}, a_{\mathrm{I}}\right)}{\partial a_{\mathrm{I}}}\right|_{a_{\mathrm{I}}=0}$.

The Cauchy condition yields

$\frac{\partial I\left(a_{\mathrm{R}}, a_{\mathrm{I}}\right)}{\partial a_{\mathrm{I}}}=\frac{\partial J\left(a_{\mathrm{R}}, a_{\mathrm{I}}\right)}{\partial a_{\mathrm{R}}}$.

Thus the lowest order approximation yields

$a_{\mathrm{I}} \simeq-I\left(a_{\mathrm{R}}, 0\right)\left(\frac{\partial J\left(a_{\mathrm{R}}, 0\right)}{\partial a_{\mathrm{R}}}\right)^{-1}$.

Then

$J\left(a_{\mathrm{R}}, 0\right)=4 \pi \sum e^{2} \mathrm{P} \int \frac{\frac{\partial f_{0}}{\partial p_{\|}}}{v_{\|}-a_{\mathrm{R}}} \mathrm{d}^{3} p$

where $P$ denotes the Cauchy principal value, while

$I\left(a_{\mathrm{R}}, 0\right)=4 \pi^{2} \sum e^{2} \int \frac{\partial f_{0}}{\partial p_{\|}} \delta\left(v_{\|}-a_{\mathrm{R}}\right) \mathrm{d}^{3} p$.

Using the notation of Pohl \& Schlickeiser (2000) the initial distribution function in the blast wave frame is (after correction of a typo in their Eq. (3))

$f(\boldsymbol{p}, t=0)=\frac{n_{\mathrm{i}} \delta\left(p_{\perp}\right) \delta\left(p_{\|}+P\right)}{2 \pi p_{\perp}}+\frac{n_{\mathrm{b}} \delta\left(p_{\perp}\right) \delta\left(p_{\|}\right)}{2 \pi p_{\perp}}$.

In this case Eq. (6) reduces to

$k^{2}=\Omega^{2}\left[\frac{1}{a^{2}}+\frac{\delta}{(a+V)^{2}}\right]$

where $\Omega^{2}=\omega_{\mathrm{p}, \mathrm{p}}^{2}+\omega_{\mathrm{p}, \mathrm{e}}^{2}$ and $\delta=n_{i} /\left(n_{\mathrm{b}} \Gamma^{3}\right)$.

\subsection{Properties of the dispersion relation}

A plot of $k^{2}$ versus $a$ is given in Fig. 2. To be noted from the figure is that there are always two real values of $a$, occurring in $a>0$ and $a<-V$, respectively. In the region $-V \leq a \leq 0$ the dispersion relation returns two real positive values of $a$, provided $k^{2}$ exceeds a minimum value, $k_{\text {min }}^{2}$, given by

$k_{\min }^{2}=\frac{\Omega^{2}}{V^{2}}\left(1+\delta^{1 / 3}\right)^{3}$

occurring on

$a_{\min }=-V\left(1+\delta^{1 / 3}\right)^{-1}>-V$.

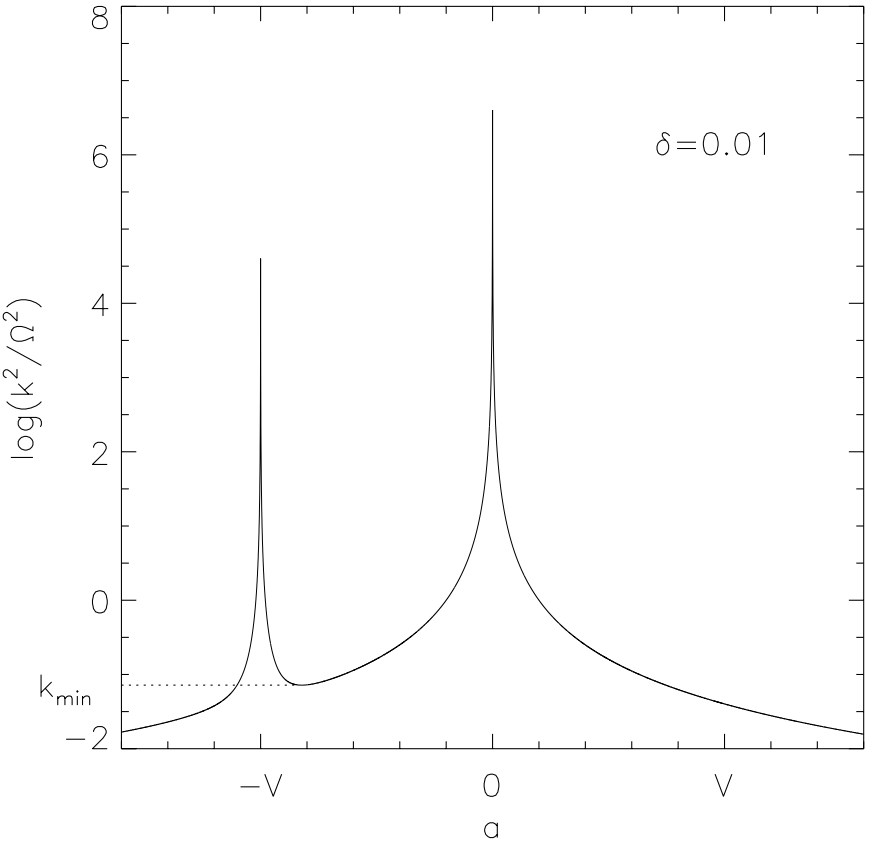

Fig. 2. The dispersion relation for longitudinal waves. Note that there is always a real solution in the range $a \leq-V$, and another real solution in $a \geq 0$. In the remaining range $-V \leq$ $a \leq 0$ the dispersion relation returns two real values of $a$ only if $\bar{k}^{2}$ exceeds $k_{\min }^{2}$.

For values $k^{2}<k_{\min }^{2}$, the solutions are complex conjugate solutions in $-V \leq a_{\mathrm{R}} \leq 0$. Note that on $k=0$, the complex solutions are

$a_{0}=-V(1+\delta)^{-1}(1 \pm \imath \sqrt{\delta})=a_{\max }(1 \pm \imath \sqrt{\delta})$.

For more general values of $k^{2}<k_{\min }^{2}$, the general solution to the dispersion relation is given through

$a V^{-1}=-\sin ^{2} \phi+\imath y_{\mathrm{I}}$

with

$\frac{k^{2}}{k_{\min }^{2}}=\left(\frac{\tan ^{2} \phi+1}{\tan ^{2} \phi-1}\right)^{2} \frac{(\tan \phi-\sqrt{\delta})(1-\tan \phi \sqrt{\delta})}{\tan \phi\left(1+\delta^{1 / 3}\right)^{3}}$

and

$y_{\mathrm{I}}^{2}=\frac{\tan \phi\left(\sqrt{\delta} \tan ^{3} \phi-1\right)}{\left(1+\tan ^{2} \phi\right)^{2}(\tan \phi-\sqrt{\delta})}$.

The range of $\phi$ is restricted to

$\delta^{-1 / 6} \leq \tan \phi \leq \delta^{-1 / 2}$.

On $\tan \phi=\delta^{-1 / 6}$ note that $k^{2}=k_{\min }^{2}$ and $a_{\mathrm{R}}=a_{\min }$, and on $\tan \phi=\delta^{-1 / 2}, k=0$ and $a_{\mathrm{R}}=a_{\max }$. Thus the range (21) covers the complete wavenumber spectrum where instability can occur. In Fig. 3 we show an enlargement of the dispersion relation in the phase velocity range where instability occurs. It is obvious that $k^{2}$ is basically independent of the phase velocity $a_{\mathrm{R}}$, unless $a_{\mathrm{R}}$ is very close to $a_{\max }$. Therefore the Taylor expansion to first order 


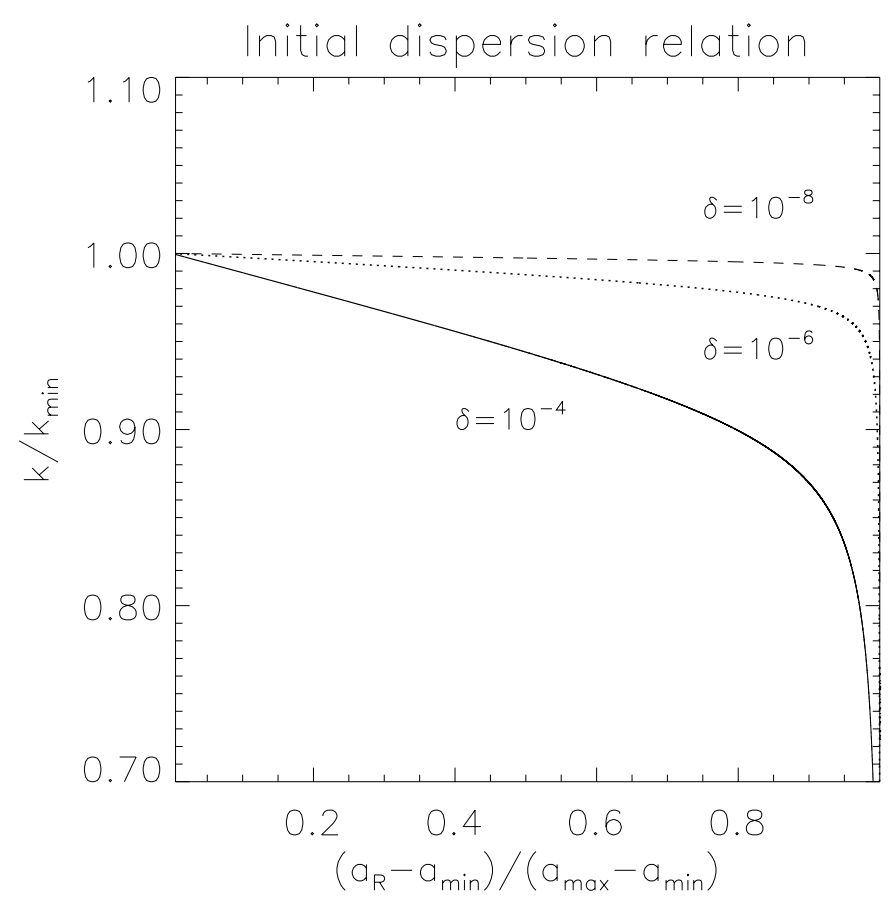

Fig. 3. An enlargement of the dispersion relation for longitudinal waves in the phase velocity range where instability occurs. For small perturbation parameters, $\delta, k / k_{\min }$ is essentially flat, unless $a_{\mathrm{R}}$ is very close to $a_{\max }$.

(Eq. (10)) is a poor approximation, for $\frac{\partial J\left(a_{\mathrm{R}}, 0\right)}{\partial a_{\mathrm{R}}} \simeq 0$. A second order expansion would provide us only with $a_{\mathrm{R}}^{2}$ which we have already derived in Eq. (20). However, Eq. (10) is very useful for determining the sign of the growth rate. Noting that $a_{\mathrm{R}}<0$, we see that $I\left(a_{\mathrm{R}}, 0\right)$ is negative for negative $\frac{\partial f_{0}}{\partial p_{\|}}$because $\frac{\partial v_{\|}}{\partial p_{\|}}>0 . J\left(a_{\mathrm{R}}, 0\right)$ is given in Eq. (14), provided $a$ is replaced by $a_{\mathrm{R}}$. Obviously $\frac{\partial J\left(a_{\mathrm{R}}, 0\right)}{\partial a_{\mathrm{R}}}<0$, and thus $a_{\mathrm{I}}<0$. Since $k<0$ we get for the growth rate

$\gamma=k a_{\mathrm{I}}=|k|\left|a_{\mathrm{I}}\right|>0$.

As in the non-relativistic case parallel electrostatic waves grow as long as the distribution function is inverted, whereas relativistic Landau damping would occur for $\frac{\partial f_{0}}{\partial p_{\|}}>0$ with $p_{\|}<0$.

The square of the growth rate is

$\gamma^{2}=k^{2} V^{2} y_{\mathrm{I}}^{2}=\Omega^{2} \frac{\left(\sqrt{\delta} \tan ^{3} \phi-1\right)(1-\tan \phi \sqrt{\delta})}{\left(\tan ^{2} \phi-1\right)^{2}}$.

In Figs. 4 and 5 we show the growth rate as a function of the wavenumber $k$ and phase velocity, $a_{\mathrm{R}}$, respectively. The growth rate is sharply peaked at wavenumbers close to $k_{\min }$, but is essentially independent of the phase velocity, $a_{\mathrm{R}}$.

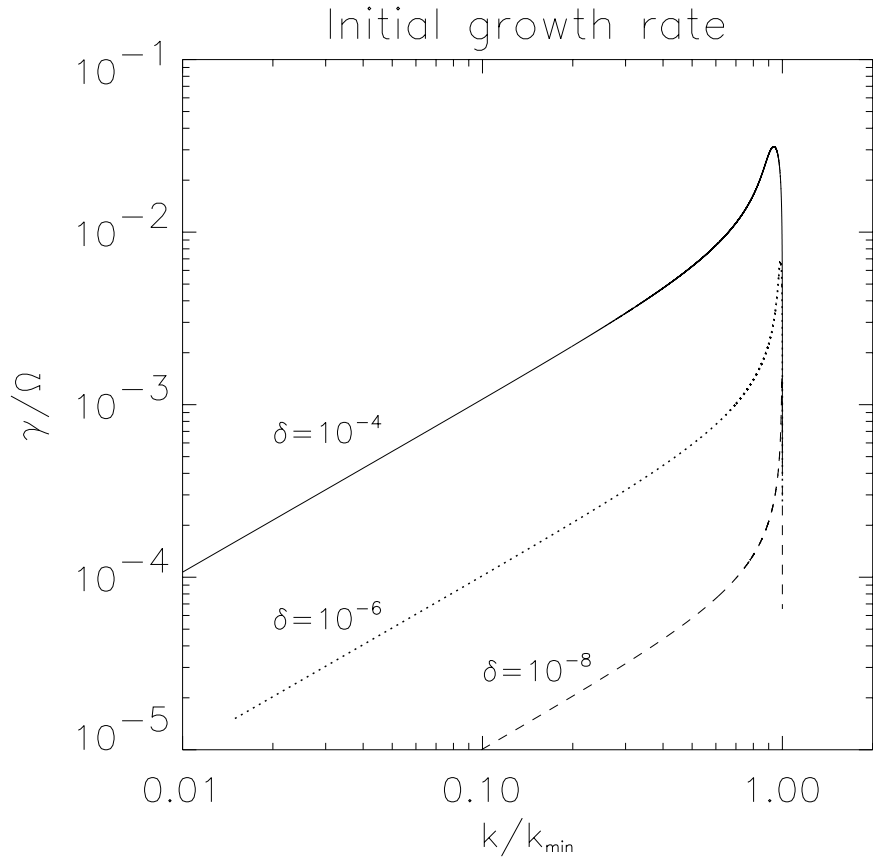

Fig. 4. The growth rate $\gamma$ as a function of the wave number $k$ for three values of the perturbation parameter $\delta=n_{i} /\left(n_{\mathrm{b}} \Gamma^{3}\right)$. Most of the instability occurs in a small wavenumber range close to $k_{\text {min }}$.

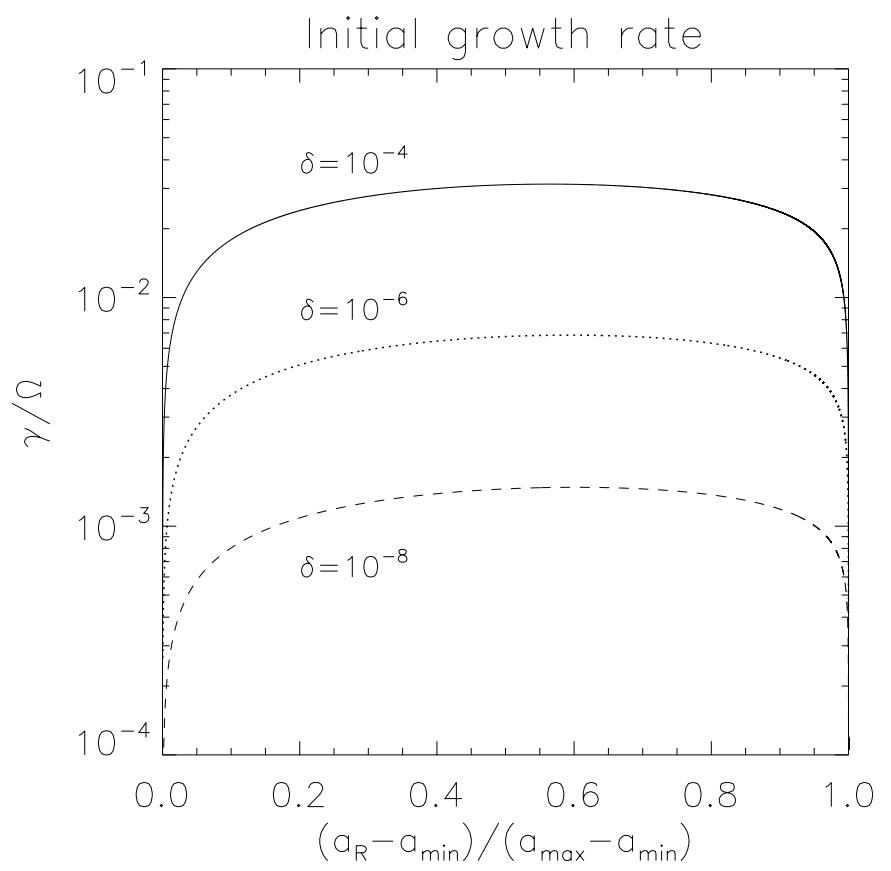

Fig. 5. The growth rate $\gamma$ as a function of the phase velocity $a_{\mathrm{R}}$ for three values of the perturbation parameter $\delta$. The perturbation parameter controls only the speed of the instability, not its spectral form.

\subsection{Quasi-linear behavior}

The time-dependent behavior of the intensities $I(k, t)$ of the excited waves is given by (Lerche 1967; Lee \& Ip 1987)

$\frac{\partial}{\partial t} I(k, t)=2 \gamma I(k, t)$ 
Note that instability occurs only for negative wave phase speeds $a_{\mathrm{R}}$, implying negative wavenumbers $k$.

To describe the long-term influence of the excited waves on the beam particles, which are the particles that can resonate with the waves, one uses the quasi-linear Fokker-Planck equation. Because the longitudinal waves act with an electric vector only, and because that vector parallels the magnetic field, the phase space density for the resonant particles then has only its momentum parallel to the ambient field influenced by the longitudinal turbulence. Hence, the corresponding Fokker-Planck equation reads

$$
\frac{\partial f}{\partial t}=\frac{\partial}{\partial p_{\|}}\left(D \frac{\partial f}{\partial p_{\|}}\right)
$$

where the diffusion coefficient, $D$, is given by

$$
\begin{aligned}
D & =\frac{\left\langle\Delta p_{\|}^{2}\right\rangle}{\Delta t}=16 \pi^{2} e^{2} \int_{k_{\min }}^{0} \mathrm{~d} k I(k) \delta\left[k\left(v_{\|}-a_{\mathrm{R}}\right)\right] \\
& =16 \pi^{2} e^{2} \int_{k_{\min }}^{0} \mathrm{~d} k \frac{I(k)}{|k|} \delta\left(v_{\|}-a_{\mathrm{R}}\right) .
\end{aligned}
$$

The corresponding diffusion equation for each resonant particle species is

$$
\frac{\partial f}{\partial t}=16 \pi^{2} e^{2} \frac{\partial}{\partial p_{\|}}\left(\int_{k_{\min }}^{0} \mathrm{~d} k \frac{I(k)}{|k|} \delta\left(v_{\|}-a_{\mathrm{R}}\right) \frac{\partial f}{\partial p_{\|}}\right)
$$

where $a_{\mathrm{R}}$ is related to the wavenumber $k$ through Eq. (19).

Note that some care has to be exercised in treating the $\delta$-function in Eq. (27) for two reasons. First, slight variations in $a_{\mathrm{R}}\left(v_{\|}\right)$correspond to large variations in $p_{\|}$ for relativistic particles. Second, during the evolution of the system the initial beam may disperse, for example through interactions with electromagnetic turbulence, so that a range of $p_{\|}$would correspond to a given $v_{\|}$. We consider here two extreme cases:

(1) when the variation in phase speed is much larger than the variation in transverse speed;

(2) when the variation in transverse speed is much larger than the variation in phase speed.

Consider each case in turn.

\subsection{Highly variable phase speed}

In this situation one can effectively set the resonant particles to a cold gas approximation and deal only with the bulk flow.

One conventionally argues that the wave generation is sufficiently rapid that the local (in time) wave intensity has reached a quasi-steady state in the sense that it alters quasi-adiabatically as the particles change their bulk streaming parameters to feed the waves.

Because of the unknown nature of the initial wave spectrum, and because other processes ignored in the development will also influence the generation of waves, one of the standard devices is to ignore the wave intensity spectrum generation and to use models of how one believes the waves spectrum has evolved to its current state. This particular decoupling of the wave and particle behaviours has been an enormous success in cosmic ray astrophysics in general and in heliospheric physics (a thorough review can be found in Schlickeiser 2001). Under this scenario one writes Eq. (26) now with $I(k, t)$ a wave intensity spectrum to be prescribed by the user.

In addition, the parameters related to the relaxation of the plasma, $V, a_{\mathrm{R}}$, etc., are considered to be independent of time in evaluating the diffusion coefficient (26), but are then allowed to evolve with time once the diffusion coefficient is evaluated in order that the long term evolution of the plasma distribution function can be tracked.

The particle velocities, $v_{\|}$, for which resonance can be established, are those which satisfy

$\frac{-V}{1+\delta} \leq v_{\|} \leq \frac{-V}{1+\delta^{1 / 3}}$

corresponding to particle momenta, $p_{\|}$, in the range

$\frac{-V \Gamma}{\sqrt{1+\delta \Gamma^{2}(2+\delta)}} \leq \frac{p_{\|}}{m} \leq \frac{-V \Gamma}{\sqrt{1+\delta^{1 / 3} \Gamma^{2}\left(2+\delta^{1 / 3}\right)}}$.

Clearly, the range of resonant particle momenta is sensitive to $2 \delta \Gamma^{2}$ and $2 \delta^{1 / 3} \Gamma^{2}$. If both are small compared to unity then the range of resonant momenta is close to the initial momentum of the beam particles, $-P$, and has a width of $\sim P \delta^{1 / 3} \Gamma^{2}$, while, if $2 \delta \Gamma^{2}$ and $2 \delta^{1 / 3} \Gamma^{2}$ are both large compared to unity then the particle momentum range is far from $-P$ and has a width of $\sim P / \sqrt{2 \delta \Gamma^{2}}$. Thus the quasi-linear evolution is sensitive to the values of $2 \delta \Gamma^{2}$ and $2 \delta^{1 / 3} \Gamma^{2}$, both of which are required to be small compared to unity in the astrophysical application to AGN (Pohl \& Schlickeiser 2000).

When we consider the growth rate (Eq. (23)) as a function of phase velocity, we see in Fig. 5 that the growth rate is essentially independent of $a_{\mathrm{R}}$. Neglecting the possible effects of damping and cascading, we can therefore expect that the intensity spectrum in phase velocity, $I\left(a_{\mathrm{R}}\right)=I(k) \frac{\partial k}{\partial a_{\mathrm{R}}}$ is flat between $a_{\min }$ and $a_{\max }$ and zero outside this range (Akhiezer et al. 1975). Upon interaction with this wave spectrum the beam will relax to a plateau distribution between the velocities $-V$ and $v_{\|}=a_{\min }$. The energy available for the build-up of the waves comes from the beam. Because the wave growth is much faster than the relaxation of the beam, we may estimate the final wave intensity spectrum as the ratio of the energy lost by the beam during relaxation to a plateau between the velocities $-V$ and $v_{\|}=a_{\text {min }}$ and the phase velocity range in which effective growth occurs, namely $\delta^{1 / 3} V$. Therefore we may write

$\Delta E \simeq \frac{n_{i}}{2} m_{\mathrm{p}} c^{2} \Gamma^{3} \delta^{1 / 3}$

$\Delta a_{\mathrm{R}} \simeq V \delta^{1 / 3}$

$I\left(a_{\mathrm{R}}\right) \simeq \frac{\Delta E}{\Delta a_{\mathrm{R}}} \simeq \frac{n_{i} m_{\mathrm{p}} c^{2} \Gamma^{3}}{2 V}=I_{0}$ 
where we keep in mind that $a_{\mathrm{R}}$ is to be taken between $a_{\min }$ and $a_{\max }$. The diffusion coefficient is then

$$
\begin{aligned}
D\left(p_{\|}\right) & =16 \pi^{2} e^{2} I_{0} \int_{a_{\max }}^{a_{\min }} \mathrm{d} a_{\mathrm{R}} \frac{1}{|k|} \delta\left(v_{\|}-a_{\mathrm{R}}\right) \\
& =16 \pi^{2} e^{2} \frac{I_{0}}{\left|k\left(a_{\mathrm{R}}=v_{\|}\right)\right|} \\
& \simeq 2 \pi m_{\mathrm{e}} m_{\mathrm{p}} c^{2} \Gamma^{3} \Omega \frac{n_{i}}{n_{\mathrm{b}}}
\end{aligned}
$$

where we have used again that $k \simeq k_{\min } \simeq-V / \Omega$ in the resonant range. The time scale for the relaxation of the beam by the electrostatic instability can then be estimated as

$\tau \simeq \frac{P^{2}}{D} \simeq \frac{m_{\mathrm{p}} n_{\mathrm{b}}}{2 \pi m_{\mathrm{e}} n_{i} \Omega \Gamma} \simeq 5 \times 10^{-3} \frac{\sqrt{n_{\mathrm{b}}}}{\Gamma n_{i}} \mathrm{~s}$.

In term of the interstellar matter density in the laboratory frame, $n_{i}^{*}$, and the scaled variables $n_{8}=10^{-8} n_{\mathrm{b}}$ and $\Gamma_{2}=10^{-2} \Gamma$ the relaxation time scale reads

$\tau=5 \times 10^{-3} \frac{\sqrt{n_{8}}}{\Gamma_{2}^{2} n_{i}^{*}} \quad \mathrm{~s}$

which is two orders of magnitude shorter than the time scale for the electromagnetic instability derived in Pohl \& Schlickeiser (2000).

\subsection{Highly variable transverse speed}

Suppose the initial beam is slightly dispersed, such that all particles have the same total momentum, $P$, but instead of flying exactly backwards $(\mu=-1)$ the particles homogeneously occupy a fixed solid angle element defined by $\mu \leq \mu_{\mathrm{c}}$. This initial condition approximates the situation after the onset of pitch angle scattering but long before the isotropization is completed. The initial distribution function in the blast wave frame is then

$$
\begin{aligned}
f(\boldsymbol{p}, t=0)= & \frac{n_{\mathrm{b}} \delta\left(p_{\perp}\right) \delta\left(p_{\|}\right)}{2 \pi p_{\perp}} \\
& +\frac{n_{i} \delta\left(\sqrt{p_{\perp}^{2}+p_{\|}^{2}}-P\right) \Theta\left(\mu_{\mathrm{c}}-\frac{p_{\|}}{P}\right)}{2 \pi P^{2}\left(1+\mu_{\mathrm{c}}\right)} .
\end{aligned}
$$

The dispersion relation (6) becomes

$k^{2}=\Omega^{2}\left[\frac{1}{a^{2}}+\frac{\delta \Gamma^{2}}{c^{2}\left(1+\mu_{\mathrm{c}}\right)} \int_{-1}^{\mu_{\mathrm{c}}} \mathrm{d} y \frac{\beta^{-2}-y^{2}}{\left(y-\frac{a}{V}\right)^{2}}\right]$

where $\beta=V / c, y=p_{\|} / P$, and $\delta=n_{i} /\left(n_{\mathrm{b}} \Gamma^{3}\right)$ as in Eq. (17). The integral in Eq. (37) cannot be trivially solved and we obtain the dispersion relation for a dispersed beam with $x=a / V$ and $\epsilon=1+\mu_{\mathrm{c}}$

$$
\frac{k^{2} V^{2}}{\Omega^{2}}=\frac{1}{x^{2}}+\delta \Gamma^{2} g(x)
$$

with

$g(x)=\frac{1}{\epsilon} \int_{-1}^{\epsilon-1} \mathrm{~d} y \frac{\beta^{-2}-y^{2}}{\left(y-\frac{a}{V}\right)^{2}}$

which for $\epsilon \rightarrow 0$ would reduce to Eq. (14). For small $\epsilon$ we can derive modifications

$x=x_{0}+x_{1}$

to the cold beam solutions $x_{0}$ (Eq. (18)) of the dispersion relation by expanding the right-hand-side of Eq. (38), $\operatorname{RHS}(x, \epsilon)$.

$$
\begin{aligned}
\frac{k^{2} V^{2}}{\Omega^{2}}= & \left.\epsilon \frac{\partial \operatorname{RHS}\left(x_{0}, \epsilon\right)}{\partial \epsilon}\right|_{\epsilon=0}+\left.x_{1} \frac{\partial \operatorname{RHS}(x, 0)}{\partial x}\right|_{x=x_{0}} \\
& +\left.\frac{x_{1}^{2}}{2} \frac{\partial^{2} \operatorname{RHS}(x, 0)}{\partial x^{2}}\right|_{x=x_{0}}+\operatorname{RHS}\left(x_{0}, 0\right)
\end{aligned}
$$

The second order expansion in $x$ is necessary because the first order term in $x$ is zero for $\tan \phi=\delta^{-1 / 6}$.

The term $\operatorname{RHS}\left(x_{0}, 0\right)$ is identical to the right-hand-side of the dispersion relation for an ideal beam (14), and hence equal to $k^{2} V^{2} / \Omega^{2}$. The remaining binomial expression in $x_{1}$ is solved by

$x_{1}=\left(1+x_{0}\right) \frac{\left(1+x_{0}^{-1}\right)^{3}+\delta-\sqrt{f\left(x_{0}, \epsilon\right)}}{3\left[\left(1+x_{0}^{-1}\right)^{4}+\delta\right]}$

where

$$
\begin{aligned}
f\left(x_{0}, \epsilon\right)= & {\left[\left(1+x_{0}^{-1}\right)^{3}+\delta\right]^{2} } \\
& -3 \epsilon \delta \Gamma^{2} \frac{1+\beta^{2} x_{0}}{1+x_{0}}\left[\left(1+x_{0}^{-1}\right)^{4}+\delta\right] .
\end{aligned}
$$

Here we have also used

$\lim _{\epsilon \rightarrow 0} x_{1}=0$

to resolve the ambiguity in sign.

For the expansion we require

$\frac{\epsilon}{|1+x|} \ll 1$

which leads to a limit for the opening half-angle of the beam $\theta=-\pi+\arccos \mu_{\mathrm{c}}$

$\epsilon \simeq \theta^{2} \ll \delta^{1 / 4} \tan ^{-\frac{1}{2}} \phi=\delta^{1 / 2}$ for $\tan \phi=\delta^{-1 / 2}$.

The limiting beam opening half-angle is about 10 arcmin for $\delta=10^{-10}$. For all $\epsilon$ satisfying Eq. (45) the parallel velocities $v_{\|}$are always larger than the phase velocities in the range where instability occurs.

In Fig. 6 we show the phase velocity modification, $V \Re x_{1}$, for different parameters. The phase velocity modification scales approximately linearly with $\epsilon$, unless $a_{\mathrm{R}}$ is close to $a_{\min }$, which reflects the dominance of the first order term in $x_{1}$ over the second order term in Eq. (40) for all $a_{\mathrm{R}}$ not too close to $a_{\text {min }}$. Figure 7 shows the growth rate modification, $\gamma_{1}$, for different parameters.

In Fig. 8 we show the modified growth rate, $\gamma+\gamma_{1}$, as a function of the modified phase velocity, $a_{\mathrm{R}}+V \Re x_{1}$. To 


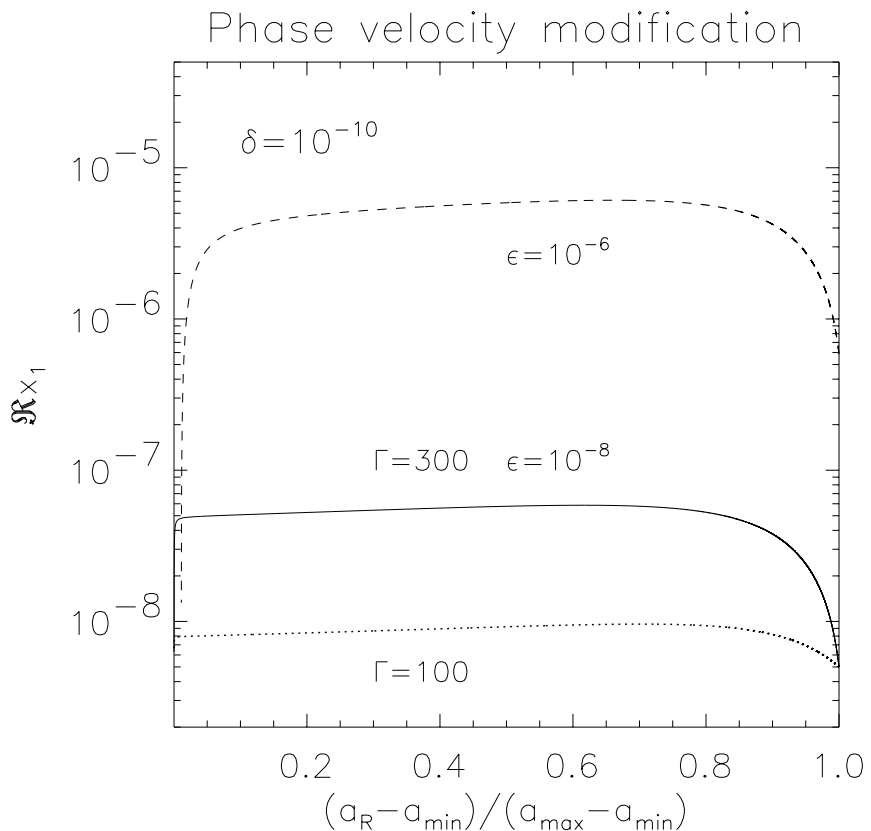

Fig. 6. The normalized phase velocity modification, $\Re x_{1}$, for different beam openings, $\epsilon$, and different bulk Lorentz factors, $\Gamma$. The perturbation parameter $\delta$ is held constant. The phase velocity modification scales approximately linearly with $\epsilon$, unless $a_{\mathrm{R}}$ is close to $a_{\mathrm{min}}$.

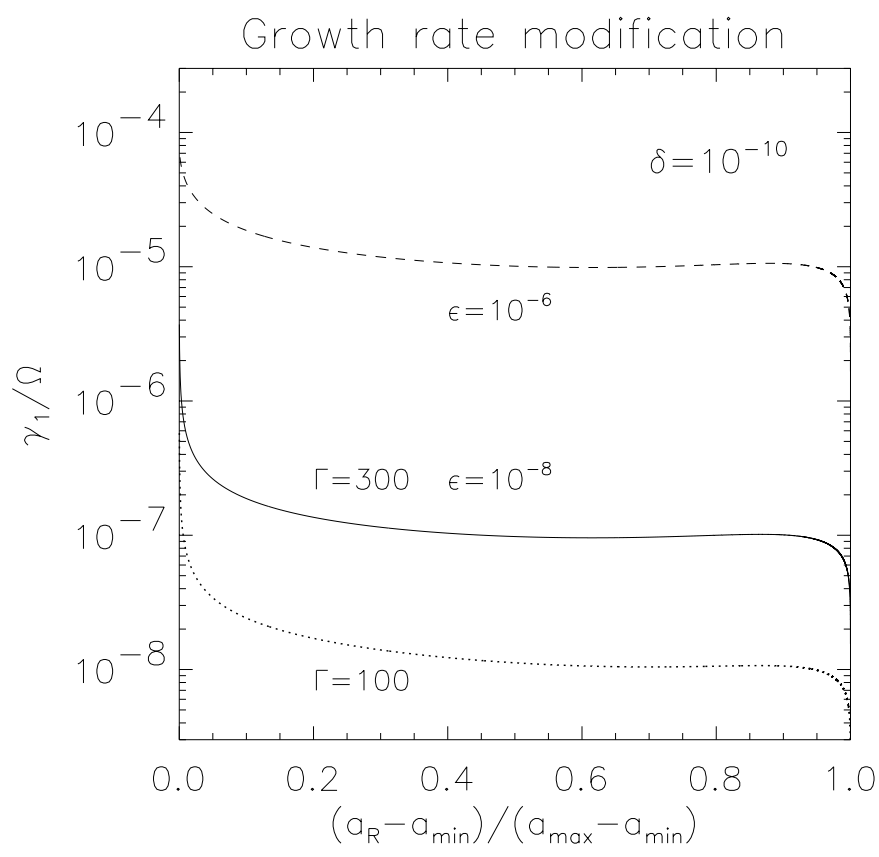

Fig. 7. The growth rate modification, $\gamma_{1}$, as a function of the unmodified phase velocity $a_{\mathrm{R}}$ for different beam openings, $\epsilon$, and different bulk Lorentz factors, $\Gamma$. The perturbation parameter $\delta$ is held constant. The growth rate modification has a similar dependence on the parameters as has the phase velocity modification.

be noted from the figure are

- the growth rate increases if the beam has a finite opening angle;

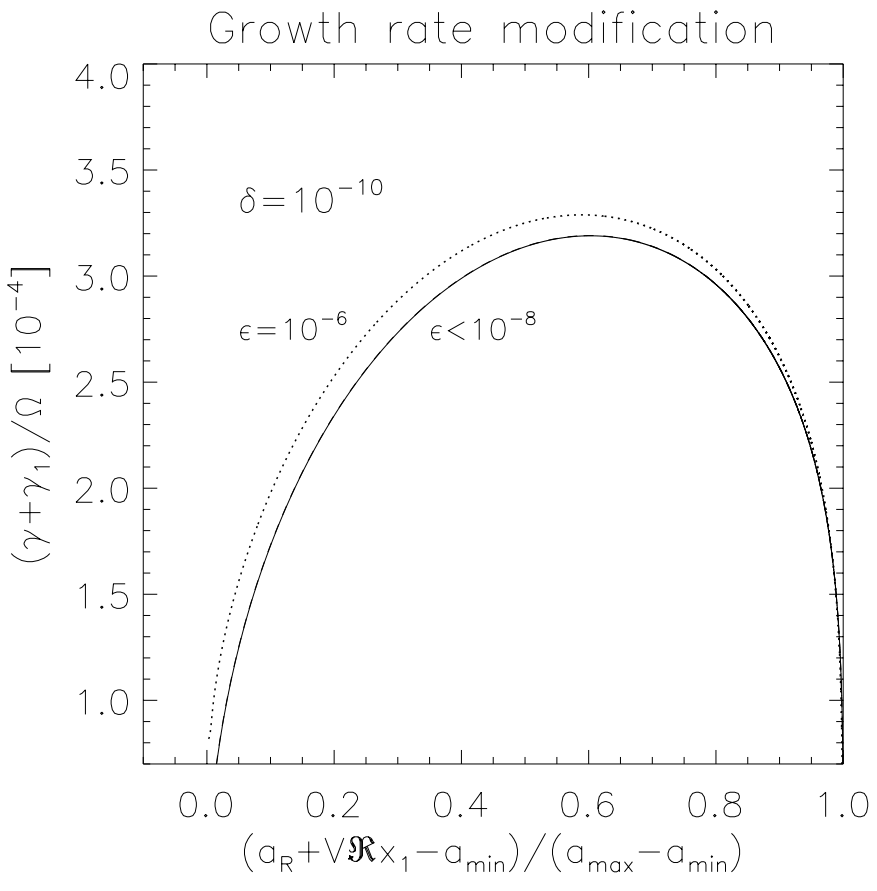

Fig. 8. The modified growth rate, $\gamma+\gamma_{1}$, as a function of the modified phase velocity, $a_{\mathrm{R}}+V \Re x_{1}$, for different beam openings, $\epsilon$. The perturbation parameter $\delta$ and the bulk Lorentz factor $\Gamma=300$ are held constant. Note that this plot has a linear scaling in units of $10^{-4}$. The solid line applies for all $\epsilon \leq 10^{-8}$, for which the modification has no visible effect. The dotted line represents the enhanced growth rate for $\epsilon=10^{-6}$.

- the phase velocity range where instability occurs is only marginally extended to phase velocities smaller than $a_{\min }$.

\section{Discussion}

We have calculated the excitation of longitudinal, electrostatic turbulence subsequent upon the injection of a relativistic electron-proton beam into a cold background plasma. In contrast to electromagnetic waves, which scatter the particles in pitch angle but preserve their kinetic energy until the distribution is isotropized, the electrostatic waves change the particles' energy until a plateau distribution is established. Our results can be applied to models of AGN and GRB, which are based on a relativistic blast waves traversing the ambient medium.

As one example of such models, two of us have recently published a calculation of an electromagnetic two-stream instability in a channeled relativistic outflow, a jet, and shown that the isotropization of the incoming interstellar particles would provide relativistic particles in the AGN jets, whose high energy emission has characteristics typical of BL Lacertae objects (Pohl \& Schlickeiser 2000). The calculations presented here expand on these results. We have shown that for parameters suitable to explain the high energy emission spectra of AGN, the electrostatic instability 


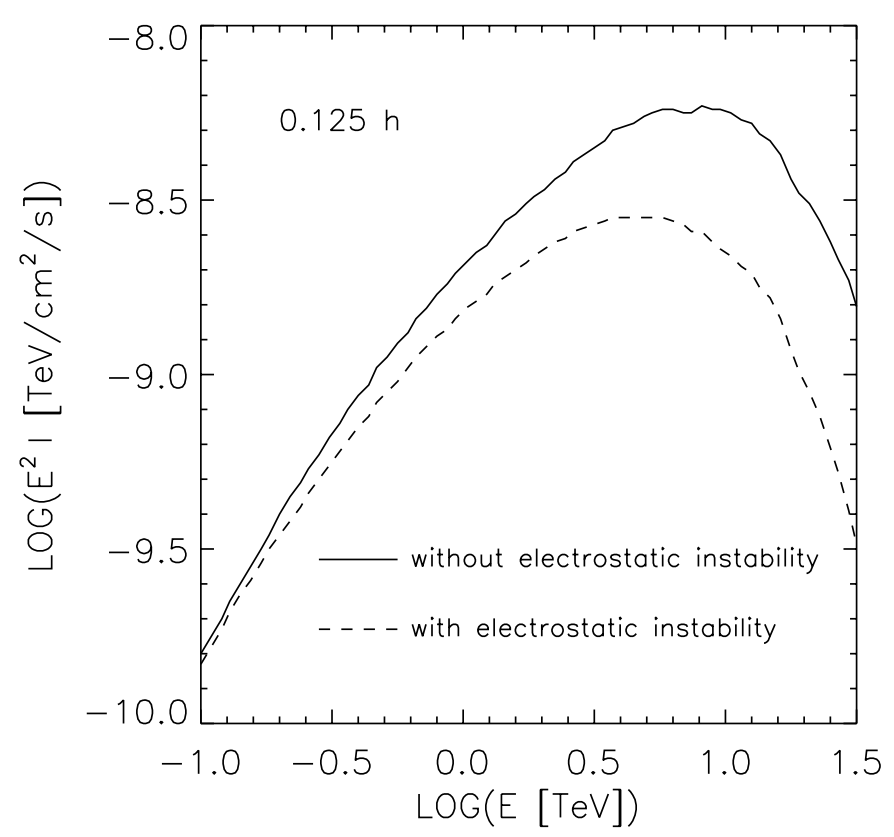

Fig. 9. $\pi^{0}$-decay spectra calculated with and without the electrostatic instability. The spectra refer to a observed time of 0.125 hours after the blast wave ran over an isolated cloud. At this time the spectrum of swept-up particles has evolved very little. At the peak, about $50 \%$ of the beam particle energy is lost due to the effect of the electrostatic instability. There is little effect at smaller photon energies.

is much faster than the electromagnetic instability, even in case of a weak dispersion of the incoming beam. The beam will therefore relax to a plateau-distribution in $p_{\|}$, which is, however, still unstable with respect to electromagnetic waves. The spectrum of electromagnetic waves excited by a plateau-distribution in $p_{\|}$will not be a $k^{-2}$ spectrum as in the case of a cold beam, for it can be understood as a superposition of the wave spectra produced by cold beams with different energies, each of which would be a $k^{-2}$ spectrum with energy-dependent upper and lower limits in $k$. Nevertheless, isotropization in the collimated blast wave would still occur on roughly the same time scale as calculated by Pohl \& Schlickeiser (2000).

About half of the energy flux carried by the incoming beam is channeled into electrostatic turbulence. It is instructive to calculate the group velocity of the waves with which the wave energy is transported. In Fig. 10 we show the group velocity for the initial ideal beam (Eq. (19)) in the phase velocity range where instability occurs. The group velocity is fairly independent of the weighted beam intensity, $\delta$, and it is always larger than $0.5 \mathrm{~V} \simeq 0.5 \mathrm{c}$. Therefore, the energy carried by the electrostatic waves is rapidly transported toward the backside of the blast wave.

Therefore, the main effect of the electrostatic instability is a change in the spectrum of swept-up particles.

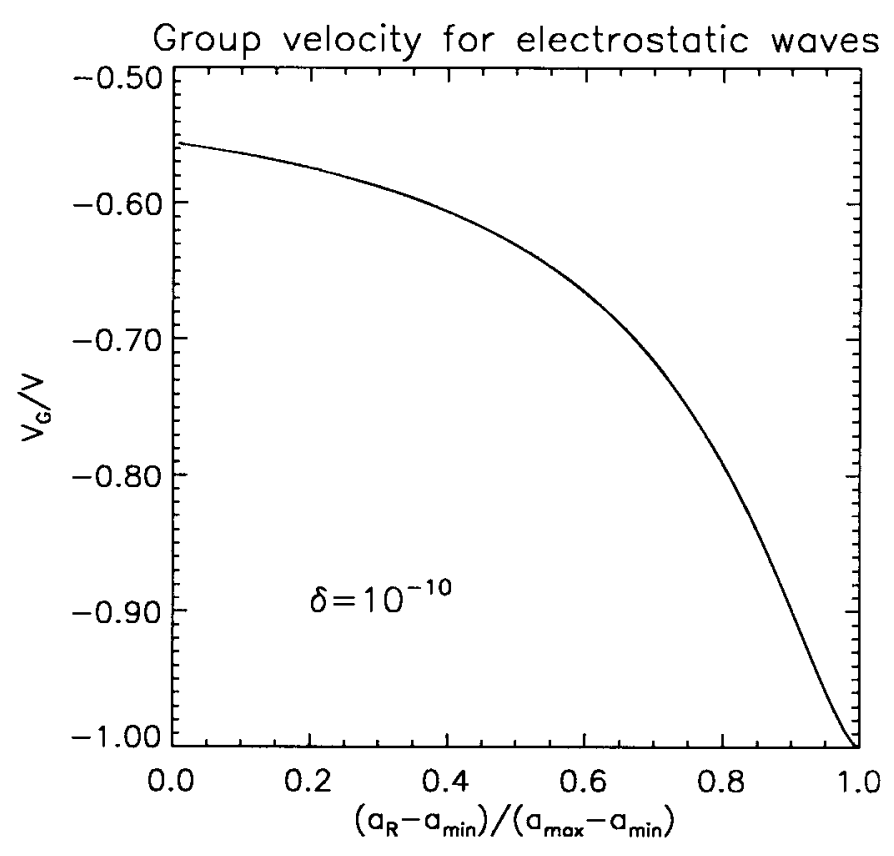

Fig. 10. The group velocity of electrostatic waves as a function of phase velocity for the initial ideal beam. The group velocity is always between about $50 \%$ and $100 \%$ of the beam velocity, which result is fairly independent of the weighted beam intensity, $\delta$.

Instead of the prior result for the differential sweep-up rate in the absence of the electrostatic instability

$\left.\dot{N}(\gamma)\right|_{\text {no e.s.i. }}=N_{0} \delta(\gamma-\Gamma)$

we now obtain

$\left.\dot{N}(\gamma)\right|_{\text {e.s.i. }}=\frac{N_{0}}{\Gamma-1} \Theta(\Gamma-\gamma)$.

In the radiation spectra this change corresponds to a reduction of efficiency by roughly $50 \%$ at the highest energies and little change at smaller energies, because in the inelastic pp collisions the pion source power scales roughly with the kinetic energy of the incident proton, hence the pion spectra are dominated by the pions produced by the protons of high energy for flat proton spectra. In Fig. 9 we show the spectra of $\pi^{0}$-decay $\gamma$-rays for the case of a fast electrostatic instability (Eq. (47)) in comparison with the case of the electromagnetic instability only (Eq. (46)). At photon energies well below the peak energy of the $\nu F_{\nu}$-spectra the difference in spectral index is only $\Delta s \simeq 0.1$. Similarly, small changes can be expected for the injection spectra of secondary electrons and hence for the synchrotron spectra at optical to X-ray frequencies. Therefore the isotropization and radiation conclusions drawn by Pohl \& Schlickeiser (2000) are still valid. Because the waves carry $50 \%$ of the energy flux of the incoming beam with a transport velocity of $50 \%-100 \%$ of 
the beam velocity, the energy densities in the final wave field and the initial beam should be similar.

$U_{\mathrm{W}}=\int \mathrm{d} a_{\mathrm{R}} I\left(a_{\mathrm{R}}\right) \simeq n_{i} m_{\mathrm{p}} c^{2} \Gamma=n_{i}^{*} m_{\mathrm{p}} c^{2} \Gamma^{2}$.

The high energy density of waves has two important aspects, which are briefly discussed in the following sections.

\subsection{Nonlinear evolution of the wave field}

Quasi-linear theory is not valid under all physical conditions. When the energy density of the wave field is not small, nonlinear effects may become important.

One important effect is nonlinear Landau damping (Sagdeev \& Galeev 1969). This is the interaction between thermal particles and the beat of two electrostatic waves: if the phase velocity of the beat wave is almost equal to the thermal velocity of charged particles in the background plasma, the particles would Landau-damp the beat wave and hence be heated. If the remaining wave has a superluminal phase velocity, it will not resonate with the beam particles anymore. As a consequence, the number of scattering processes between the beam particles and the waves, which are responsible for the production of a plateau in the beam distribution function, would be drastically reduced. This process reduces the wave intensity in the resonant phase velocity range as long as the intensity of waves with superluminal phase velocity is not so strong that nonlinear interactions in this regime cause wave energy to be retransferred to resonant subluminal phase velocities.

The nonlinear Landau damping is a process resulting from weak turbulence theory: although waves interact, the wave energy is small enough that individual waves are identifiable. If the wave energy is too high, strong turbulence theory applies (Zakharov 1972). The charge distribution in the background plasma, which results from the electric field of the waves (essentially the RHS of Eq. (4)), becomes so strong that it affects the dispersion relation. As a result, the wave field will collapse into cavitons (Goldman 1984) in which the plasma has a low density. This effect will depend on the temperature of the background plasma, and on whether or not the diffusive particle motion can effectively smear out the depleted cavitons, and thus impede their build-up.

Without having performed a detailed study of these effects in a background medium with possibly time-variable temperature caused by heating processes, we can only speculate on the impact of the nonlinear effects, which would increase the time scale for the plateau production of the beam (Eq. (35)). However, because that time scale is more than two orders of magnitude shorter than the time scale for the electromagnetic instability, even if important such nonlinear effects would likely still permit the production of a plateau on a timescale much faster than the isotropization.

\subsection{Where does the wave energy go?}

Part of the wave energy will be transferred to the background plasma in the blast wave. We have already discussed the nonlinear Landau damping, in which thermal particles would absorb the beat of two electrostatic waves. But, in the general case, the natural width of the resonance Lorentz profile combined with the Maxwellian distribution function allows thermal particles to Landau damp the waves with $v_{\phi} \simeq c$, albeit with a small probability.

We do not know the fraction of the wave energy density that is channeled into heating processes of the blast wave plasma. Here we concentrate on a qualitative discussion of the possible effects.

The wave energy is transported quickly toward the backside of the collimated blast wave. At the transition between the blast wave and its wake, the dispersive properties of the blast wave change. We can, therefore, expect that a part of the wave field is reflected at the backside, and possibly again reflected at the front side and so forth. If the reflection coefficient is not small, the wave energy density inside the blast wave would increased, until nonlinear effects at the front and backside cause the waves to escape. The heating rate would be similarly enhanced.

What would be the effect of strong heating in the blast wave? As an extreme example consider that the full wave energy flux is available for homogeneously heating the blast wave. The heating rate then is

$\dot{T} \simeq \frac{\left(\gamma_{\mathrm{a}}-1\right) n_{i}^{*} m_{\mathrm{p}} c^{3} \Gamma^{2}}{2 n_{\mathrm{b}} d k} \simeq 10^{6} \frac{n_{i}^{*} \Gamma_{2}^{2}}{n_{8} d_{13}} \mathrm{~K} / \mathrm{s}$

where we have used the scaled variables $n_{8}=10^{-8} n_{\mathrm{b}}$, $d_{13}=10^{-13} d$, and $\Gamma_{2}=10^{-2} \Gamma$. This heating rate would be too large to be balanced by any cooling process of a non-relativistic plasma. Therefore run-away heating would occur until non-thermal cooling processes set in at semirelativistic energies.

The existence of radio galaxies implies that the collimation of AGN jets is well maintained on linear scales of hundreds of kiloparsecs. A semi-relativistic plasma jet could hardly remain collimated on these scales, because the expansion by internal pressure alone would cause a substantial opening of the jet. We may take the observed collimation of jets in radio galaxies as empirical evidence against excessive heating. For the fate of the electrostatic wave field, this observation indicates that the reflection efficiency of waves at the backside of the blast wave is small, whatever the cause.

Since the waves carry momentum, even a small reflection would transfer momentum to the background plasma at the backside of the blast wave, which would then "boil off" the blast wave and fill its wake. The waves that have escaped the blast wave would fill the wake and the regions around it, and be finally damped by the interstellar plasma in the host galaxy of the AGN, thus giving rise to additional heating of the interstellar medium around the AGN jet. 
Acknowledgements. Partial support by the Verbundforschung, grant DESY-05AG9PCA, is gratefully acknowledged.

\section{References}

Akhiezer, A. I., Akhiezer, I. A., Polovin, R. V., Sitenk, A. G., \& Stepanov, K. N. 1975, Plasma Electrodynamics, vol. 2 (Oxford University Press)

Bicknell, G. V., Wagner, S. J., \& Groves, B. 2001, in High Energy Gamma-Ray Astronomy, ed. Aharonian \& Völk, AIP Conf. Proc., 558, 261

Boksenberg, A., Macchetto, F., Albrecht, R., et al. 1992, A\&A, 261,393

Catanese, M., \& Weekes, T. C. 1999, PASP, 111, 1193

Gallant, Y. A., \& Achterberg, A. 1998, Proc. of the 16th European Cosmic Ray Symposium, ed. J. Medina (University of Alcalá Press), 253
Goldman, M. V. 1984, Rev. Mod. Phys., 56, 709

Hartman, R. C., Collmar, W., von Montigny, C., \& Dermer, C. D. 1997, in Proceedings of the Fourth Compton Symposium, ed. Dermer, Strickman, \& Kurfess, AIP Conf. Proc., 410, 307

Meisenheimer, K., Röser, H.-J., \& Schlötelburg, M. 1996, A\&A, 307, 61

Mücke, A., Pohl, M., Reich, P., et al. 1996 A\&AS, 120, C541

Lee, M. A., \& Ip, W.-H. 1987, J. Geoph. Res., 92, 11041

Lerche, I. 1967, ApJ, 147, 689

Pohl, M., \& Schlickeiser, R. 2000, A\&A, 354, 395

Sagdeev, R. Z., \& Galeev, A. A. 1969, Nonlinear Plasma Theory (New York: Benjamin)

Schlickeiser, R. 2001, Cosmic Ray Astrophysics (Springer, Heidelberg)

Schuster, C., Pohl, M., \& Schlickeiser, R. 2002, A\&A, submitted

Zakharov, V. E. 1972, Sov. Phys. JETP, 35, 908 